\title{
GRUPO DE ESTUDO E APOIO DIDÁTICO COMO FORMA DE INCENTIVO À PRÁTICA DOCENTE EM ANATOMIA HUMANA
}

\author{
Raphael Lopes Olegário ${ }^{1}$ \\ ORCID: 0000-0001-8931-9533 \\ Polyanne Junqueira Silva Andresen Strini ${ }^{2}$ \\ ORCID: 0000-0003-1305-4801 \\ Paulinne Junqueira Silva Andresen Strini ${ }^{3}$ \\ ORCID: 0000-0002-1788-1014
}

Resumo: As atividades de extensão universitária constituem caminhos relevantes para permitir a formação acadêmica completa do estudante, integrando teoria e prática em uma relação bilateral com a sociedade, permitindo o diálogo e aprendizado mútuo entre ambas. $\mathrm{O}$ objetivo da presente ação de extensão foi realizar encontros semanais entre as comunidades interna e externa da Universidade Federal de Goiás, focados em grupos de estudo e discussão, incentivo a prática docente em Ciências Morfológicas, aprofundamento teórico e prático do conteúdo, além de explorar a abordagem da Anatomia Humana em concursos e processos seletivos e sua aplicabilidade no mercado de trabalho. As atividades de extensão acadêmica se

1 Bolsista de Iniciação Científica, do Departamento de Processos Psicológicos Básicos do Instituto de Psicologia (PPB/IP), Universidade de Brasília (UnB). Endereço: Campus Universitário Darcy Ribeiro, ICC Sul, Brasília, DF, Brasil. CEP 70910-900. Graduando em Educação Física (FEF/UnB) E-mail: (rlounb@gmail.com).

2 Professora Doutora de Anatomia Humana, da Unidade Acadêmica Especial de Ciências da Saúde CISAU, Curso de Medicina, da Universidade Federal de Goiás - UFG, Regional Jataí, Jataí, GO, Brasil. Endereço: Rua Rio Preto, 178, Lídice, Uberlândia - MG, CEP 38400-09o. Graduação em Odontologia/ UFU; Mestre e Doutora em Biologia Buco-Dental/UNICAMP. E-mail: (polyjsas@gmail.com).

3 Professora Doutora de Anatomia Humana do Instituto de Ciências Biomédicas - ICBIM, da Universidade Federal de Uberlândia - UFU,Uberlândia, MG, Brasil. Endereço: Rua Rio Preto, 178, Lídice, Uberlândia - MG, CEP 3840o-09o. Graduação em Odontologia/UFU; Mestre em Reabilitação Oral/ UFU; Doutora em Biologia Buco-Dental/UNICAMP. E-mail: (paulinnejsas@gmail.com). 
mostraram enriquecedoras para todos os envolvidos, os participantes tiveram a oportunidade de explorar e desenvolver na prática o que aprendem no curso universitário e o incentivo à docência em ciências morfológicas contribuiu consideravelmente para formação acadêmica, em especial, no campo da Anatomia Humana.

Palavras-chave: Anatomia. Ensino. Aprendizagem. Educação. Cadáver.

\title{
STUDY GROUPS AND TEACHING SUPPORT AS A WAY OF ENCOURAGING THE TEACHING PRACTICE IN HUMAN ANATOMY
}

\begin{abstract}
The activities of university extension are relevant ways to allow the student's complete academic formation, integrating theory and practice in a bilateral relationship with society, allowing the dialogue and mutual learning between both. The objective of this extension action was to hold weekly meetings of the internal and external community of the Federal University of Goias, focusing on study and discussion groups, encouraging the teaching practice in Morphological Sciences, theoretical and practical deepening of the content, and exploring the approach of Human Anatomy in contests and selective processes and their applicability in the labor market. The activities of academic extension were enriching for all involved, the participants had the opportunity to explore and develop in practice what they learn in the university course and the encouragement of teaching in morphological sciences has contributed considerably to academic formation, especially in the field of Human Anatomy.
\end{abstract}

Keywords: Anatomy. Teaching. Learning. Education. Cadaver.

\section{GRUPO DE ESTUDIO Y APOYO DIDÁCTICO COMO FORMA DE INCENTIVO LA PRÁCTICA DOCENTE EN ANATOMÍA HUMANA}

Resumen: Las actividades de extensión universitaria constituyen caminos relevantes para permitir la formación académica completa del estudiante, integrando teoría y práctica en una relación bilateral con la sociedad, permitiendo el diálogo y aprendizaje mutuo entre ambos. El objetivo de la presente acción de extensión fue realizar encuentros semanales de la comunidad interna y externa de la Universidad Federal de Goiás, enfocados en grupos de estudio y discusión, incentivo a la práctica docente en Ciencias Morfológicas, profundización teórica y práctica del contenido, además de explorar el abordaje de la Anatomía Humana en concursos y procesos selectivos y su aplicabilidad en el mercado de trabajo. Las actividades de extensión académica se mostraron enriquecedoras para todos los involucrados, los participantes tuvieron la oportunidad de explorar y desarrollar en la práctica lo que aprenden en el curso universitario y el incentivo a la docencia en ciencias morfológicas contribuyó considerablemente a la formación académica, en especial, en el campo de la educación Anatomía Humana.

Palabras claves: Anatomía. Enseñanza. Aprendizaje. Educación. Cadáver.

Submetido em: 24/06/2019.

Aceito em: 01/10/2019. 


\section{INTRODUÇÃO}

Uma das principais funções sociais da Universidade é a de contribuir na busca de soluções para os graves problemas sociais da população, subsidiando a formulação de políticas públicas participativas e emancipadoras. Poucos são os que possuem acesso direto aos conhecimentos gerados na universidade pública. A extensão universitária é imprescindível para a democratização do acesso a esses conhecimentos, assim como para o redimensionamento da função social da própria universidade (MENDONÇA; SILVA, 2002).

As atividades de extensão universitária constituem caminhos relevantes para permitir a formação acadêmica completa do estudante, integrando teoria e prática em uma relação bilateral com a sociedade, permitindo o diálogo e aprendizado mútuo entre ambos (MANCHUR et al., 2013). Dessa maneira, ações de extensão favorecem a construção e a socialização do conhecimento, por meio de metodologias capazes de estimular e potencializar a formação da equipe executora e do público alvo das ações.

As ações de extensão universitária podem ser úteis em permitir a formação e capacitação técnica científica adequada e de qualidade a estudantes, profissionais e indivíduos interessados em temas da área da saúde. Ações que focam na Anatomia Humana são capazes de permitir o aprofundamento teórico e prático no conteúdo ministrado e contribuir na formação do acadêmico, melhorando seu desempenho didático e incentivando a prática docente. Desta maneira, o desenvolvimento de projetos de aprimoramento nas atividades teórico e prática e a busca por novas metodologias auxiliares de ensino são importantes em promover suporte educacional e enriquecimento intelectual à comunidade acadêmica e os envolvidos, fornecendo subsídios para o conhecimento do corpo humano (COLTRO et al., 2007).

Com isso, o objetivo da presente ação de extensão foi realizar encontros semanais da comunidade interna e externa da Universidade Federal de Goiás, focados em grupos de estudo e discussão, incentivo a prática docente em Ciências Morfológicas, aprofundamento teórico e prático do conteúdo, além de explorar a abordagem da Anatomia Humana em concursos e processos seletivos e sua aplicabilidade no mercado de trabalho. 


\section{METODOLOGIA}

Foram realizadas oficinas de estudo com exposição teórica e prática de conteúdos referentes à Anatomia Humana com embasamento na necessidade de aprendizagem de tópicos relevantes para formação acadêmica em cursos da área da saúde, além de enfoque na preparação do acadêmico para realização de avaliações variadas. Ressalta-se que o conteúdo de Anatomia Humana está presente em provas de diversos processos seletivos, como por exemplo, concursos militares, residências médicas e multiprofissionais, concursos para professores, dentre outros.

As oficinas foram realizadas com frequência semanal com duração de 90 minutos, uma vez por semana, em sala de aula com recursos audiovisuais quando necessário e em laboratório de Anatomia Humana com acesso a peças cadavéricas. As oficinas foram divididas em tópicos que abrangeram todos os sistemas anatômicos do corpo humano. Seguiu-se a padronização de exposição teórica dos conteúdos em um primeiro momento e discussão em grupos em um segundo momento.

O incentivo à prática docente se deu pelo fato dos alunos realizarem as oficinas/ aulas para outros alunos e também o esclarecimento de dúvidas dos mesmos. No momento das oficinas, os alunos faziam o papel de professor explicando os conteúdos propostos para outros estudantes, sempre com a supervisão de um docente da área. Como instrumento de acompanhamento do progresso dos participantes durante o desenvolvimento das ações, observou-se o engajamento e participação nas atividades ao longo de todas as oficinas propostas. Ademais, ao fim de cada oficina se pedia o feedback a respeito da eficácia das atividades propostas e opiniões para melhoria das próximas ações.

\section{RESULTADOS}

O enfoque das oficinas foi nos sistemas biológicos no qual se elaboraram palestras teóricas e práticas em laboratório, além de exercícios oriundos de processos seletivos (concursos públicos e residências médicas e/ou multiprofissionais). Houve grupos de estudo individualizados e em grupo para discussão de temáticas mais específicas em que os estudantes apresentaram maior dificuldade.

A relação de oficinas teóricas se encontra no Quadro 1 e oficinas práticas com peça cadavérica na Quadro 2. Participaram das atividades propostas estudantes vinculadas a cursos de graduação em Educação Física, Medicina, Nutrição, Física Médica, Farmácia, Ciências Biológicas e Biotecnologia da Universidade Federal 
de Goiás cadastrados no projeto de extensão universitária PJ400-2017, além de estudantes de outras instituições de ensino.

QUADRO 1: RELAÇÃO DE TEMAS DAS OFICINAS TEÓRICAS.

\section{OFICINAS TEÓRICAS EM SALA DE AULA/AUDITÓRIO}

Noções básicas do Sistema Cardiovascular e suas generalidades

Noções básicas do Sistema Respiratório e suas generalidades

Noções básicas do Sistema Digestório e suas generalidades

Noções básicas do Sistema Nervoso e suas generalidades

Noções básicas do Sistema Endócrino e suas generalidades

Noções básicas do Sistema Geniturinário e suas generalidades

Noções básicas do Sistema Esquelético e suas generalidades

Noções básicas do Sistema Imunológico e suas generalidades

Noções básicas do Sistema Tegumentar e suas generalidades

FONTE: ELABORADO PELOS AUTORES.

\section{QUADRO 2: RELAÇÃO DE TEMAS DAS OFICINAS PRÁTICAS.}

\section{AULAS PRÁTICAS EM LABORATÓRIO}

Demonstração do coração e suas estruturas a partir de peças molhadas

Demonstração das Veias e Artérias e suas estruturas a partir de peças molhadas

Demonstração dos componentes do Sistema Respiratório e suas estruturas a partir de peças molhadas

Demonstração do Trato Gastrointestinal Superior e suas estruturas a partir de peças molhadas Demonstração do Trato Gastrointestinal Inferior e suas estruturas a partir de peças molhadas

Demonstração do Sistema Nervoso Central e suas estruturas a partir de peças molhadas

Demonstração do Sistema Nervoso Periférico e suas estruturas a partir de peças molhadas

Demonstração do Sistema Nervoso aplicado a Traumatologia

Demonstração do Sistema Urinário e suas estruturas a partir de peças molhadas

Demonstração do Sistema Reprodutor Feminino e suas estruturas a partir de peças molhadas

Demonstração do Sistema Reprodutor Masculino e suas estruturas a partir de peças molhadas

Demonstração do Esqueleto Axial e suas estruturas a partir de peças molhadas

Demonstração do Esqueleto Apendicular e suas estruturas a partir de peças molhadas

Demonstração do Sistema Esquelético aplicado a Traumatologia

Demonstração do Sistema Muscular e suas estruturas a partir de peças molhadas

Demonstração do Sistema Muscular aplicado a Ciência do Esporte

FONTE: ELABORADO PELOS AUTORES. 
As atividades realizadas foram publicadas em formato de trabalho científico por meio de apresentação em congresso de extensão e pesquisa acadêmica, permitindo o contato dos participantes com a divulgação científica no âmbito de eventos científicos para incentivo de publicação em modalidade de pôster.

\section{DISCUSSÃO}

Atividades no âmbito da extensão universitária são relevantes para formação profissional de acadêmicos da área da saúde (CALIPO, 2009). Tais procedimentos podem ser uma ferramenta para estimular a prática docente e o aprimoramento do conteúdo acadêmico, preparando o indivíduo para o mercado de trabalho e promovendo a contextualização do saber específico inserido no futuro profissional. Com isso, a atuação de graduandos em ações de formação continuada para estudantes e profissionais da área da saúde, com vistas ao aprofundamento do saber acadêmico em Anatomia Humana, é capaz de incentivar o desenvolvimento de metodologias de ensino-aprendizagem e formas diversificadas e criativas de apresentação do conteúdo, traduzindo a linguagem formal do docente, na tentativa de facilitar o entendimento e a fixação do assunto (JENIZE, 2004).

Busca também uma abordagem voltada à importância da anatomia na formação do aluno e sua aplicabilidade no cotidiano profissional e no mercado de trabalho. Neste contexto, discutir a formação dos estudantes, de novos docentes e profissionais apresenta-se como uma prática reflexiva bastante relevante. Em concordância com Gonçalves et al. (2005), trata-se de um campo específico de intervenção profissional capaz de contribuir no desenvolvimento intelectual do ser humano. Reflexões coletivas e o estabelecimento de um grupo focal podem ser úteis em demonstrar a importância do maior preparo e apoio aos novos docentes, a necessidade de troca de experiências e a sistematização de vivências anteriores, além da necessidade de gerar mais espaços e formas de reflexões sobre as práticas docentes. (SANTOS; ALMEIDA FILHO, 2012)

Assim, as reflexões se iniciam quando se começa a investigar e a questionar, estando ligadas ao processo de pesquisa. Em associação, o grupo focal pode ser empregado com o objetivo de identificar problemas e avaliar conceitos em uma discussão específica, sendo utilizado como ferramenta avaliativa em pequenos grupos (CAPLAN, 1990). Com isso, a formação e produção do conhecimento, envolvendo professores e alunos de forma dialógica (Figura 1), se constituem parte integrante da dinâmica pedagógica capaz de permitir uma flexibilidade curricular que possibilite a formação crítica. 


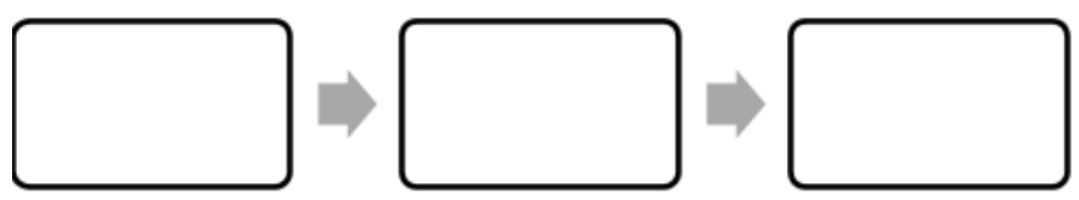

FIGURA 1: FLUXO DE FORMAÇÃO E PRODUÇÃO DE CONHECIMENTOS DE FORMA DIALÓCICA. FONTE: AUTORES (2019)

Neste sentido, os vários procedimentos metodológicos vislumbrados na literatura podem ser adequados aos estudantes de graduação, curso técnico e profissional, por meio de ações de extensão universitária, a fim de permitir sua formação e capacitação técnica científica adequada e de qualidade. As atividades desenvolvidas permitiram o aprimoramento do conhecimento de conteúdos da Anatomia Humana aplicados em diversos campos do saber de forma contextualizada com as necessidades de formação profissional de estudantes da área da saúde e correlatas. Segundo Nunes et al. (2011), essas novas ferramentas conduzem à melhoria de desempenho do aluno, já que facilitam o aprendizado de conteúdos considerados difíceis de compreender.

A ação oportunizou o contato do estudante com a docência no âmbito do ensino superior, incentivando-o a prática da docência. Ademais, houve oficinas práticas realizadas em laboratório de Anatomia Humana que permitiram o contato direto com peça cadavérica. No ensino e aprendizagem de conteúdos da disciplina Anatomia é de fundamental importância a observação direta das estruturas tridimensionais nas peças cadavéricas (INFANTOSI et al., 2000).

Diversas ferramentas podem ser utilizadas no aprimoramento do processo ensino-aprendizagem, com destaque para a aplicabilidade da Anatomia Humana na formação do aluno de graduação, de cursos técnicos e profissionais da área da saúde e na utilização do conteúdo na vida pós-universidade. Assim, grupos de discussão, oficinas interativas, cursos, aulas, monitorias, debates e palestras sobre conteúdos específicos da área, resolução de questões utilizadas em processos seletivos, concursos e pós-graduação, aplicação de simulados, elaboração de perguntas, resolução de exercícios são mecanismos capazes de promover a integração do conteúdo com a necessidade dos envolvidos (PERRENOUD, 1993). Além disso, dinâmicas, métodos de memorização, exposição e solução de dúvidas, charadas, jogos, atividades artísticas, testes online, criação de blogs e sites, e o uso de demais ferramentas digitais podem atender a demanda dos participantes, auxiliar no enfrentamento das dificuldades e na compreensão da aplicação do conteúdo fora do contexto acadêmico. 
Adicionalmente, material didático e apostilas foram confeccionadas permitindo o estudo e contribuindo no aprendizado e fixação dos temas abordados. Tais metodologias permitem auxiliar na aquisição do conhecimento para alunos em geral e principalmente aqueles de baixo rendimento, estimulando o estudo e facilitando a fixação e aprofundamento do assunto (LIB NEO, 1994). Visa garantir o aprendizado e êxito na formação do estudante e incentivar a criação de práticas didáticas diferenciadas e contextualizadas com a realidade, além de simplificar o processo ensino aprendizagem e estimular a prática docente, trazendo benefícios tanto para a equipe executora quanto para o público alvo.

Dessa forma, destaca-se a importância do conteúdo de anatomia para a formação do aluno e para sua atuação profissional, traduzindo o conteúdo da linguagem formal do professor para o aluno, facilitando o aprendizado e trabalhando as diversas temáticas de forma interdisciplinar.

\section{CONCLUSÃO}

As atividades de extensão acadêmica se mostraram enriquecedoras para todos os envolvidos, os participantes tiveram a oportunidade de explorar e desenvolver na prática o que aprendem no curso universitário. $\mathrm{O}$ incentivo à docência em Ciências Morfológicas contribuiu consideravelmente para formação acadêmica, em especial, no campo da Anatomia Humana. Diante do exposto, é notório a importância de projetos de extensão para a formação acadêmico/profissional de alunos.

\section{REFERÊNCIAS}

CALIPO, D. Projetos de extensão universitária crítica: Uma ação educativa transformadora. Campinas, 2009. Base de dados do Scielo. Disponível em: http://www.itcp.unicamp.br/drupal/files/Projetos\%20de\%20extensao\%20 universitaria_\%20Daniel\%20Bortolotti.pdf. Acesso em: 12 jun. 2019.

CAPLAN, S. Using focus group methodology for ergonomic design. Ergonomics, v. 33, n. 5, p. 527-33, 1990.

COLTRO, A. F.; LAAT, E. F.; SANTOS, R. G. O projeto de extensão: "da escola à universidade" na cidade de Irati. Revista Mackenzie de Educação Física e Esporte, v.6, n. 2, p. 185-189. 2007. 
GONÇALVES, H. A. Manual de metodologia da pesquisa. São Paulo: Avercamp, 2005.

INFANTOSI A. F. C., KLEMT A. Visualização 3D da dissecção crânio humano: A surface method for visualising the $3 \mathrm{D}$ dissection of the human skull. Rev Bras Eng Biom v. 1, n. 1, p. 21-37. 2000.

JENIZE, E. As Práticas Curriculares e a Extensão Universitária. 2004. Disponível em: http://br.monografias.com/trabalhos-pdf901/as-practicas-curriculares/as-practicas-curriculares.pdf. Acesso em: 10 jun. 2019.

LIB NEO, J. C. O processo de ensino na escola. São Paulo: Cortez, 1994. P. 77-118.

MANCHUR, J.; SURIANI,A. L. A.; CUNHA, M. C. A Contribuição de projetos de extensão na formação profissional de graduandos de licenciaturas. Revista Conexão UEPG. V. 9, n. 2 - jul./dez. 2013.

MENDONÇA, S. G. L.; SILVA, P. S. Extensão Universitária: Uma nova relação com a administração pública. Extensão Universitária: ação comunitária em universidades brasileiras. São Paulo, v. 3, p. 29-44, 2002.

NUNES E. P. S., NUNES F. L. S., TORI R. Avaliação da aquisição de conhecimento em ambientes virtuais de aprendizagem 3D para treinamento médico. Anais XXXI Congresso da Sociedade Brasileira de Computação; 2011 jul. 19-22; Natal-RN, Brasil. Disponível em: http://www.dimap.ufrn.br/csbc2011/ Anais/eventos/contents/WIM/WIM_Sessao_4_Artigo_6_Nunes.pdf. Acesso em: 19 jun. 2019.

SANTOS, F. S.; ALMEIDA FILHO, N. A quarta Missão da Universidade: Internacionalização Universitária na Sociedade do Conhecimento. 1.ed. Coimbra: 2012. 